\title{
Fuzzy logic and adaptive neuro-fuzzy inference system for characterization of contaminant exposure through selected biomarkers in African catfish
}

\begin{abstract}
This study represents a first attempt at applying a fuzzy inference system (FIS) and an adaptive neuro-fuzzy inference system (ANFIS) to the field of aquatic biomonitoring for classification of the dosage and time of benzo[a]pyrene $(\mathrm{BaP})$ injection through selected biomarkers in African catfish (Clarias gariepinus). Fish were injected either intramuscularly (i.m.) or intraperitoneally (i.p.) with BaP. Hepatic glutathione S-transferase (GST) activities, relative visceral fat weights (LSI), and four biliary fluorescent aromatic compounds (FACs) concentrations were used as the inputs in the modeling study. Contradictory rules in FIS and ANFIS models appeared after conversion of bioassay results into human language (rulebased system). A "data trimming" approach was proposed to eliminate the conflicts prior to fuzzification. However, the model produced was relevant only to relatively low exposures to $\mathrm{BaP}$, especially through the i.m. route of exposure. Furthermore, sensitivity analysis was unable to raise the classification rate to an acceptable level. In conclusion, FIS and ANFIS models have limited applications in the field of fish biomarker studies.
\end{abstract}

Keyword: Modeling; Fish biomarkers; Fuzzy inference system; Adaptive neuro-fuzzy inference system; Benzo[a]pyrene; African catfish; Clarias gariepinus 